Original paper

\title{
Preoperative alkaline phosphatase is a potential predictor of short-term outcome of surgery in infants with biliary atresia
}

\author{
Samira Abdel-Wahab Abdel-Aziz', Mostafa Mohamad Sira', Emad Hamdy Gad², Islam Ayoub², Mervat Soltan ${ }^{3}$ \\ 'Department of Pediatric Hepatology, Gastroenterology and Nutrition, National Liver Institute, Menoufia Universtiy, Egypt \\ ${ }^{2}$ Department of Hepatopancreaticobiliary Surgery, National Liver Institute, Menoufia Universtiy, Egypt \\ ${ }^{3}$ Department of Pathology, National Liver Institute, Menoufia Universtiy, Egypt
}

\begin{abstract}
Aim of the study: Biliary atresia (BA) is a fibro-inflammatory cholangiopathy of intra- and extrahepatic biliary radicles. The standard-of-care treatment is surgical restoration of bile flow by Kasai hepatoportoenterostomy (HPE). We aimed to identify the predictors of short-term outcome of the Kasai operation three months postoperatively.

Material and methods: This retrospective study included 107 infants diagnosed with BA by intraoperative cholangiography. All underwent a Kasai operation. The surgical outcome was classified after 3 months post-operatively as successful (bilirubin $\leqslant 2 \mathrm{mg} / \mathrm{dl}$ ) or failed (bilirubin $>2 \mathrm{mg} / \mathrm{dl}$ ). The two groups were compared according to basic clinical, ultrasonographic and histopathological characteristics.

Results: Of the studied patients $29(27.1 \%)$ had a successful outcome while 78 (72.9\%) had failed Kasai HPE. Of the preoperative characteristics, lower age and lower serum alkaline phosphatase (ALP) were significantly associated with successful surgical outcome ( $p=0.009$ and $<0.0001$, respectively). In addition, surgical type of BA affected the short-term outcome $(p=0.017)$, while there was no statistically significant difference regarding the other studied parameters between groups. Age of 69.5 days or less was predictive of successful outcome with $74.4 \%$ specificity but with low sensitivity (58.6\%), and ALP at a cutoff level of $532.5 \mathrm{U} / \mathrm{l}$ or less was predictive of successful outcome with $75.9 \%$ sensitivity and $74.4 \%$ specificity.

Conclusions: Younger age at the time of surgery and lower ALP are good predictors for the short-term outcome of Kasai HPE with better performance of ALP. This may help to anticipate those who can benefit from surgical correction and those who should be given high priority for transplant referral.
\end{abstract}

Key words: age, outcome, alkaline phosphatase, Kasai hepatoportoenterostomy.

Address for correspondence

Dr. Samira Abdel-Wahab Abdel-Aziz, National Liver Institute, Egypt, e-mail: samiraabdelwahab@liver.menofia.edu.eg

\section{Introduction}

Biliary atresia (BA) is a neonatal disease that is characterized by progressive fibro-inflammatory cholangiopathy usually manifesting in the first month of life. It causes severe cholestasis, rapidly progressing biliary cirrhosis and death in the first years of life, if left without treatment [1]. Although infectious, immune, genetic, and morphogenic origins have been invoked; the etiopathogenesis of BA remains unknown. This hampers preventive strategies and therapies designed to stop progression of the fibro-inflammatory process of the bile ducts [2].

The primary treatment of BA is surgical. A Kasai hepatoportoenterostomy operation (HPE) in type 3 $\mathrm{BA}$ and its modified techniques in non-type $3 \mathrm{BA}$ should be performed to restore the biliary flow to the intestine following surgical removal of extrahepatic 
remnants of the residual biliary duct. If surgery fails, or biliary cirrhosis and life-threatening complications develop, liver transplantation is then necessary, for which BA represents the most frequent indication in the pediatric age group [3].

The Kasai HPE remains the preferred initial treatment, even though it is estimated that about $80 \%$ of patients with BA will eventually need a liver transplantation. Most studies attribute the short-term success of the Kasai operation to several prognostic factors. Among them are many that are nonmodifiable such as the surgical type of BA. Other prognostic factors are described as modifiable, such as early diagnosis, a careful surgical procedure and prevention of postoperative cholangitis [3].

The aim of the current study is to identify the predictors of short-term outcome of the Kasai operation three month postoperatively.

\section{Material and methods}

\section{Study population}

This retrospective study included 107 infants with BA, all of whom underwent Kasai HPE and were followed up for three months postoperatively. At this time point, infants were divided according to the total serum bilirubin level into two groups: group $1(n=29)$ with a successful outcome (total bilirubin $\leq 2 \mathrm{mg} / \mathrm{dl}$ ) and group $2(n=78)$ with a failed outcome (total bilirubin $>2 \mathrm{mg} / \mathrm{dl}$ ) [4]. All cases were recruited from the Department of Pediatric Hepatology, Gastroenterology and Nutrition, National Liver Institute, Menoufia University, Egypt. Informed consent was obtained for experimentation from the parents. This study was approved by the Research Ethics Committee of the National Liver Institute, Menoufia University, Egypt.

\section{Etiological diagnosis}

After full history taking, thorough clinical examination, and routine investigations, the patients were allocated as having BA by an abdominal exploration; if a gallbladder was visualized and patent, the child underwent an operative cholangiogram, and this maneuver was not done if there was no gallbladder or a fibrotic gallbladder and/or an atretic extrahepatic biliary tree was present. The surgical type of BA was also identified as described by Superina et al. [5]. Routine investigations included total and direct bilirubin, total serum proteins, albumin, alanine transaminase (ALT), aspartate transaminase (AST), alkaline phosphatase (ALP), gamma-glutamyl transpeptidase (GGT), pro- thrombin time (PT), complete blood count, ultrasonography Doppler US, and liver biopsy.

\section{Liver biopsy}

Ultrasound-guided liver biopsy was performed for all patients using a Tru-Cut needle (GTA, Quistello, $\mathrm{MN}$, Italy). A core of liver tissue containing at least 5 portal tracts was considered sufficient. Liver tissue specimens were fixed in formalin and embedded in paraffin. Five- $\mu \mathrm{m}$ thick sections were cut, mounted on glass slide and stained with hematoxylin and eosin to evaluate pathological changes, with Mason-Trichrome that stains collagen fibers to assess fibrosis, and with Perls' Prussian blue stain which reveals iron deposits. Portal fibrosis and inflammatory activity were assessed using semi-quantitative histopathological scores as described by Russo et al. [6].

\section{Statistical analysis}

Descriptive results were expressed as mean \pm standard deviation (mean \pm SD) or number and percentage. For quantitative data, significance between two groups was tested by the Mann-Whitney $U$-test and significance between more than two groups was tested by the Kruskal-Wallis test. For qualitative and categorical data, significance was tested by the Chi-square test or Fisher's exact test. Correlation was tested by Spearman's test. The diagnostic performance of significant parameters was assessed by calculating the area under the receiver-operating characteristic (ROC) curve. The cutoff value for optimal clinical performance was determined from the ROC curves. The diagnostic performance was measured as sensitivity and specificity, expressed as percentages. Significance was set to $p<0.05$. Statistical analysis was performed using SPSS software version 21 (IBM Corp. IBM SPSS Statistics for Windows, Version 21.0. Armonk, NY: IBM Corp.).

\section{Results}

\section{Study population characteristics}

The current study included 107 infants, 59 male and 48 females, with BA whose ages ranged from 42 to 143 days (mean 76.78). According to surgical outcome, 29 (27.1\%) had successful Kasai while 78 (72.9\%) had failed Kasai. Comparing the basic characteristics between groups, age and ALP were significantly lower in the successful group compared to the failure group ( $p=0.009$ and $<0.0001$, respectively) while other baseline demographic, clinical, and laboratory parameters were comparable in both groups (Table 1). 


\section{Ultrasonographic findings and surgical types of biliary atresia}

As regards surgical type of BA, infants with BA type $3,84 / 107$ patients $(78.5 \%)$, significantly more often had a failed outcome three months postoperatively than non-type 3 patients $(p=0.017)$ (Table 2). Both successful and failure groups were comparable regarding the ultrasonographic findings. Contractile GB was found in 7 patients in the failed group, while the majority of patients had either non-contractile or abnormal atretic GB. Triangular cord (TC) sign was reported in $40.2 \%$ of all the study population.

\section{Histopathological findings}

At the time of liver biopsy, 10 (9.3\%) patients had absent or fibrous expansion of some portal tracts, $26(24.3 \%)$ had fibrous expansion of most portal tracts, $46(43 \%)$ had focal porto-portal bridging while 25 infants $(23.4 \%)$ had marked bridging. Comparing the two outcome groups, there was no statistically significant difference regarding the grade of fibrosis or the necroinflammatory activity or the other studied histopathological parameters (Table 3).

\section{Clinical performance of age and alkaline phosphatase in predicting successful Kasai}

Age of 69.5 days or less was predictive of successful outcome with $74.4 \%$ specificity but with low sensitivity (58.6\%) with 95\% confidence interval (95\% CI) 0.554 0.777. In addition, ALP had a better performance in predicting success of Kasai operation with $75.9 \%$ sensitivity and $74.4 \%$ specificity (95\% CI: $0.735-0.917$ ). Area under the receiver operating characteristic curve (AUROC) values were $66.5 \%$ and $82.6 \%$ respectively (Fig. 1).

\section{Discussion}

Determination of the prognosis of patients with BA remains a significant matter as early reliable prediction of prognosis of patients with BA will be helpful to plan a long-term strategy for BA treatment [7].

It was reported that there was markedly improved native liver survival without liver transplantation in children whose bilirubin normalized within 3 months postoperatively $[8,9]$.

In this study, 29 out of 107 (27.1\%) patients had successful Kasai, while the other $78(72.9 \%)$ patients had failed Kasai. In line with our results, Sangkhathat et al. [10] reported a success rate of $33.4 \%$. Similarly, Wildhaber et al. [11] reported success rates of $36 \%$.
Table 1. Demographic, clinical and laboratory characteristics according to outcome

\begin{tabular}{|c|c|c|c|}
\hline Characteristics & $\begin{array}{l}\text { Successful } \\
(n=29)\end{array}$ & $\begin{array}{c}\text { Failed } \\
(n=78)\end{array}$ & $p$ value \\
\hline Age at surgery (days) & $70.21 \pm 12.11$ & $79.23 \pm 15.93$ & 0.009 \\
\hline Male, $n(\%)$ & $15(51.7)$ & $44(56.4)$ & 0.665 \\
\hline Clay stool, $n(\%)$ & $29(100)$ & $78(100)$ & 1.0 \\
\hline Hepatomegaly, $n(\%)$ & $26(100)$ & $77(100)$ & 0.06 \\
\hline Splenomegaly, $n(\%)$ & $18(62.1)$ & $49(62.8)$ & 0.943 \\
\hline Ascites, $n(\%)$ & $0(0.0)$ & $0(0.0)$ & NA \\
\hline Total bilirubin (mg/dl) & $11.157 \pm 3.08$ & $11.16 \pm 3.82$ & 0.666 \\
\hline Direct bilirubin (mg/dl) & $7.68 \pm 2.16$ & $7.70 \pm 3.04$ & 0.708 \\
\hline $\begin{array}{l}\text { Alanine } \\
\text { transaminase (U/I) }\end{array}$ & $164.55 \pm 91.98$ & $130.28 \pm 73.36$ & 0.062 \\
\hline $\begin{array}{l}\text { Aspartate } \\
\text { transaminase (U/I) }\end{array}$ & $262.69 \pm 156.67$ & $209.67 \pm 109.48$ & 0.132 \\
\hline Albumin (g/dl) & $3.41 \pm 0.62$ & $3.6 \pm 0.51$ & 0.124 \\
\hline $\begin{array}{l}\text { Alkaline } \\
\text { phosphatase (U/I) }\end{array}$ & $410.7 \pm 177.76$ & $701.7 \pm 322.5$ & $<0.0001$ \\
\hline $\begin{array}{l}\text { Gamma glutamyl- } \\
\text { transpeptidase (U/I) }\end{array}$ & $910.17 \pm 780.36$ & $801.15 \pm 505.82$ & 0.902 \\
\hline Prothrombin time (s) & $11.81 \pm 0.78$ & $12.11 \pm 0.94$ & 0.117 \\
\hline Hemoglobin (g/dl) & $9.99 \pm 1.18$ & $10.01 \pm 1.19$ & 0.877 \\
\hline $\begin{array}{l}\text { White blood cells } \\
\left(\times 10^{3} / \mu \mathrm{l}\right)\end{array}$ & $11.74 \pm 2.95$ & $13.06 \pm 4.03$ & 0.072 \\
\hline Platelets $\left(\times 10^{3} / \mu \mathrm{l}\right)$ & $489.75 \pm 167.18$ & $467.32 \pm 166.33$ & 0.323 \\
\hline
\end{tabular}

NA - not applicable

Table 2. Ultrasonographic characteristics and surgical type of biliary atresia (BA) according to outcome

\begin{tabular}{|c|c|c|c|}
\hline Characteristics & $\begin{array}{c}\text { Successful }(n=29) \\
n(\%)\end{array}$ & $\begin{array}{c}\text { Failed }(n=78) \\
n(\%)\end{array}$ & $p$ value \\
\hline TC-sign & $10(34.5)$ & $23(42.3)$ & 0.463 \\
\hline \multicolumn{4}{|l|}{ GB contractility } \\
\hline Contractile GB & $1(3.4)$ & $7(9)$ & \multirow{3}{*}{0.608} \\
\hline Non-contractile & $11(37.9)$ & $26(33.3)$ & \\
\hline Rudimentary & $17(58.6)$ & 45 (57.7) & \\
\hline \multicolumn{4}{|l|}{ Surgical type of BA } \\
\hline Non-type 3 & $11(37.9)$ & $12(15.4)$ & \multirow{2}{*}{0.017} \\
\hline Type 3 & $18(62.1)$ & $66(84.6)$ & \\
\hline
\end{tabular}

BA - biliary atresia, TC sign - triangular cord sign, GB - gallbladder, non-type 3 - type 1 and type 2

Yet, some other studies reported higher success rates ranging from $43 \%$ to $50 \%$ [12].

Three different anatomical types of BA have been identified by the Japanese classification, according to level of obstruction of the extrahepatic bile ducts; 
Table 3. Histopathological characteristics according to outcome

\begin{tabular}{|c|c|c|c|}
\hline Characteristics & $\begin{array}{c}\text { Successful }(n=29) \\
n(\%)\end{array}$ & $\begin{array}{c}\text { Failed }(n=78) \\
n(\%)\end{array}$ & $p$ value \\
\hline \multicolumn{4}{|l|}{ Grade of liver fibrosis } \\
\hline Absent or fibrous expansion of some portal tracts & $3(10.3)$ & $7(9)$ & \multirow{5}{*}{0.283} \\
\hline Fibrous expansion of most portal tracts & $8(27.6)$ & $18(23.1)$ & \\
\hline Focal porto-portal bridging & $15(51.7)$ & $31(39.7)$ & \\
\hline Marked bridging & $3(10.3)$ & $22(28.2)$ & \\
\hline Cirrhosis & $0(0.0)$ & $0(0.0)$ & \\
\hline \multicolumn{4}{|l|}{ Portal cellular infiltrate } \\
\hline No/Minimal & $11(37.9)$ & $23(29.5)$ & \multirow{3}{*}{0.527} \\
\hline Mild & $12(41.4)$ & $40(41.4)$ & \\
\hline Moderate/Severe & $6(20.7)$ & $15(19.2)$ & \\
\hline Ductular proliferation & $29(100)$ & $76(97.4)$ & 1 \\
\hline Bile plugs & 27 (93.1) & $68(87.2)$ & 0.388 \\
\hline Multi-nucleated giant hepatocytes & $12(41.4)$ & $35(44.9)$ & 0.772 \\
\hline
\end{tabular}

A

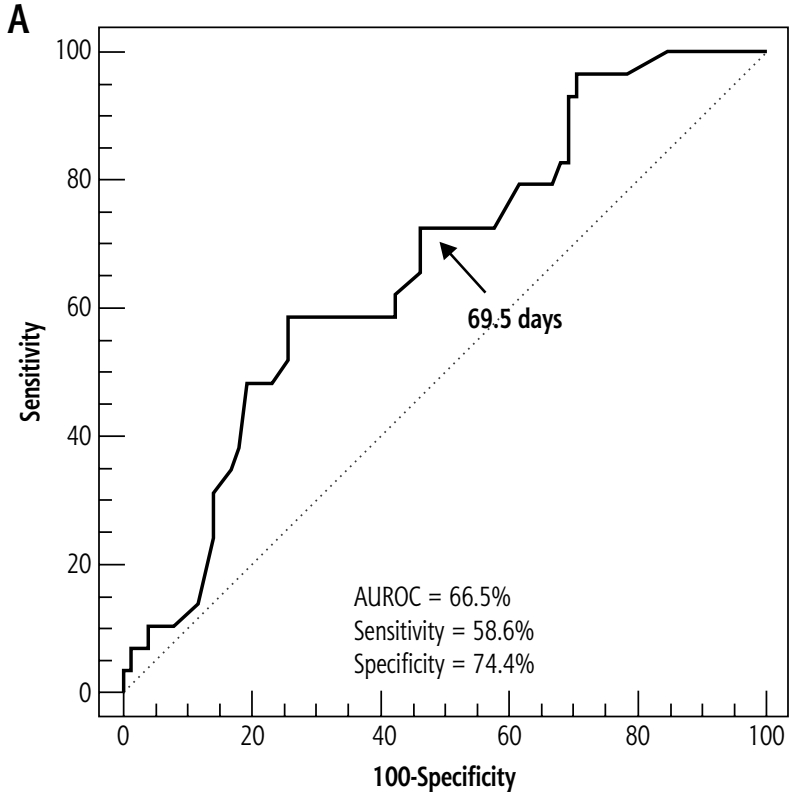

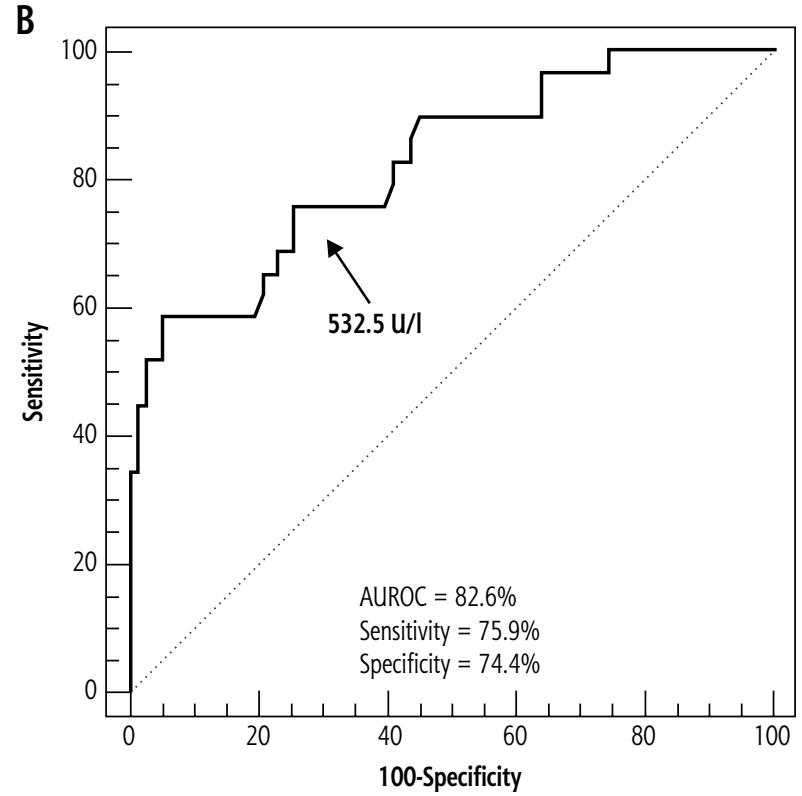

AUROC - area under receiver operating characteristic curve

Fig. 1. Performance of age (A) and alkaline phosphatase (B) in discriminating between biliary atresia (BA) patients with successful and those with failed Kasai

type 1: atresia limited to the common bile duct; type 2: atresia limited to the common bile duct and hepatic duct; type 3: atresia of the whole biliary tree with upper level of obstruction at the porta hepatis [1].

Out of the $107 \mathrm{BA}$ infants included in the study, $84(78.5 \%)$ were of type 3 . Our center's previous experience [13] revealed that type 3 atresia comprises about $90 \%$ of BA patients. This may be the cause of the low success rate in our study. Many authors have report- ed that infants with type 3 BA are usually associated with the worst post-operative outcome [14]. These data agree with our results, where infants with BA type 3 significantly more often had a failed outcome three months postoperatively than non-type 3 patients ( $p=0.017)$; however, the small number of cases with type $1 \mathrm{BA}$ ( 1 patient, $0.9 \%$ ), and type 2 BA (22 patients, $20.6 \%$ ) limits the statistical power of analysis of postoperative outcome by surgical type of BA. 
Analysis of the clinical characteristics of both outcome groups revealed no significant difference except for age at surgery, which was significantly lower in the successful group compared to the failure group $(p=0.009)$. Infant age 69.5 days or less is predictive of success with $74.4 \%$ specificity but with low sensitivity (58.6\%). This finding agrees with several studies indicating that age at surgery is a major determinant of short- and long-term outcome of the Kasai operation. Short-term results of the Kasai operation are better when it is performed during the first three months of life, with clear evidence that the earlier the operation is, the better is the outcome [15].

It was reported by Sastiono et al. [16] that the success rate was $33.3 \%$ in those operated on below the age of 90 days. Another study by Schoen et al. [17] reported a success rate of $83 \%$ of infants who were operated on before 75 days of life. These studies, in addition to the current study, disagree with Davenport et al., who found that increasing the age at operation did not adversely affect the outcome [18].

ALP is commonly monitored in clinical practice and can be elevated in various hepatobiliary disease processes. In humans, it is found in liver, bones, intestine, placenta, kidneys and leukocytes [19]. Hepatic ALP is concentrated near the biliary canalicular membrane of the hepatocyte. Cholestasis enhances the synthesis and release of ALP, and accumulating bile salts increase its release from the cell surface [20]. Concomitant elevations of ALT, AST and GGT indicate that the elevation of ALP is from a hepatic source [21]. The common causes of elevated ALP include primary sclerosing cholangitis, primary biliary cirrhosis, biliary obstruction, infiltrative diseases, and congestive hepatopathy [22]. Bastis-Maounis et al. [23] revealed that serum ALP ranged from normal to significantly elevated values in infants with biliary atresia.

Among liver function tests, ALP was the only significant variable in the current study. Lower levels of ALP were significantly associated with a good operative outcome, and a cutoff level of $532.5 \mathrm{U} / \mathrm{l}$ or less can predict successful Kasai HPE with $75.9 \%$ sensitivity and $74.4 \%$ specificity. In line with our results, Udomsinprasert et al. [24] reported a significantly lower ALP in those with a good outcome compared to those with a bad outcome ( $400.9 \pm 253.3 \mathrm{U} / \mathrm{l}$ vs. $586.7 \pm 274.9 \mathrm{U} / \mathrm{l}$; $p=0.004)$. In addition to being an indicator for the severity of biliary obstruction, ALP is likely to be a good predictor of Kasai outcome. Other studies have described higher ALP levels as a predictor of poor outcome in alcoholic hepatitis [25] and primary biliary cirrhosis [26].
In the current study, liver fibrosis was not a significant parameter in predicting the success of the Kasai operation. In line with our results, Sookpotarom et al. [12] reported the lack of impact of liver pathology on outcome. On the other hand, other studies reported that higher grades of fibrosis are associated with worse outcome [27]. Interestingly, other researchers evaluated the amount of fibrosis by computerized quantification. They found that the pattern of fibrosis as evaluated by the Ishak score [28] showed no correlation with the surgical outcome or with the amount of fibrosis as detected by collagen staining and image analysis, while the amount of fibrosis at the time of HPE was a valid marker in predicting the outcome [29]. The Russo score [6] used in our study is dependent on the pattern rather than the amount of fibrosis. This may explain the lack of significance of liver fibrosis in predicting the Kasai outcome.

Regarding the necroinflammatory activity, mild to severe necroinflammatory activity was observed in $68.2 \%(73 / 107)$ of patients, but without any statistical difference between the two groups $(p=0.527)$. Our results disagree with Hukkinen et al. [9], who detected that a high grade of portal inflammation at operation can predict clearance of jaundice ( $p=0.011)$ with age decreased from 73 days to 54 days $(p=0.014)$. Our result may be because of the older age of patients (mean 76.78 days) in the current study. Moyer et al. [30] were consistent with our suggestion. They found an association between hepatic inflammation and younger age (median age 55 days old) at diagnosis, which raised the possibility that the presence of inflammation reflects the early stages of disease and may relate to clinical outcome.

The limitation of the study is its retrospective nature and the assessment of other potential confounders that may affect the outcome such as surgical technique and postoperative medical co-morbidities were not addressed.

\section{Conclusions}

In conclusion, younger age at the time of surgery and lower ALP are good predictors for the short-term outcome of Kasai HPE with better performance of ALP. This may help to anticipate those who can benefit from surgical correction and those who should be given high priority for transplant referral.

\section{Disclosure}

Authors report no conflict of interest. 


\section{References}

1. Chardot C. Biliary atresia. Orphanet J Rare Dis 2006; 1: 28 .

2. Mack CL, Sokol RJ. Unraveling the pathogenesis and etiology of biliary atresia. Pediatr Res 2005; 57: 87R-94R.

3. Wildhaber BE. Biliary atresia: 50 years after the first Kasai. ISRN Surg 2012; 2012: 15.

4. Kelly DA, Davenport M. Current management of biliary atresia. Arch Dis Child 2007; 92: 1132-1135.

5. DeRusso PA, Ye W, Shepherd R, et al. Growth failure and outcomes in infants with biliary atresia: a report from the Biliary Atresia Research Consortium. Hepatology 2007; 46: 1632-1638.

6. Superina R, Magee JC, Brandt ML, et al. The anatomic pattern of biliary atresia identified at time of Kasai hepatoportoenterostomy and early postoperative clearance of jaundice are significant predictors of transplant-free survival. Ann Surg 2011; 254: 577-585.

7. Russo P, Magee JC, Boitnott J, et al. Design and validation of the biliary atresia research consortium histologic assessment system for cholestasis in infancy. Clin Gastroenterol Hepatol 2011; 9: 357-362.

8. Shneider BL, Brown MB, Haber B, et al. A multicenter study of the outcome of biliary atresia in the United States, 1997 to 2000. J Pediatr 2006; 148: 467-474.

9. Hukkinen M, Kerola A, Lohi J, et al. Treatment policy and liver histopathology predict biliary atresia outcomes: results after national centralization and protocol biopsies. J Am Coll Surg 2018; 226: 46-57.

10. Sangkhathat S, Patrapinyokul S, Tadtayathikom K, et al. Peri-operative factors predicting the outcome of hepatic porto-enterostomy in infants with biliary atresia. J Med Assoc Thai 2003; 86: 224-231.

11. Wildhaber BE, Coran AG, Drongowski RA, et al. The Kasai portoenterostomy for biliary atresia: a review of a 27 -year experience with 81 patients. J Pediatr Surg 2003; 38: 1480-1485.

12. Sookpotarom P, Vejchapipat P, Chittmittrapap S, et al. Shortterm results of Kasai operation for biliary atresia: experience from one institution. Asian J Surg 2006; 29: 188-192.

13. El-Guindi MA, Sira MM, Sira AM, et al. Design and validation of a diagnostic score for biliary atresia, J Hepatol 2014; 61: 116123.

14. Nightingale S, Stormon MO, Oloughlin EV, et al. Early post-hepatoporto-enterostomy predictors of native liver survival in biliary atresia. J Pediatr Gastroenterol Nutr 2017; 64: 203-209.

15. Nio M, Sasaki H, Wada M, et al. Impact of age at Kasai operation on short- and long-term outcomes of type III biliary atresia at a single institution. J Pediatr Surg 2010; 45: 2361-2363.

16. Sastiono, Maheranny M, Hanifa. Factors affecting the success rate of Kasai portoenterostomy. N Ropanasuri J Surg 2016; 1: 27-30.

17. Schoen BT, Lee H, Sullivan K, et al. The Kasai portoenterostomy: When is it too late? J Pediatr Surg 2001; 36: 97-99.

18. Davenport M, Kerkar N, Mieli-Vergani G, et al. Biliary atresia: the King's College Hospital experience (1974-1995). J Pediatr Surg 1997; 32: 479-485.

19. Wiwanitkit V. High serum alkaline phosphatase levels, a study in 181 Thai adult hospitalized patients. BMC Family Practice 2001; 2: 2 .

20. Giannini EG, Testa R, Savarino V. Liver enzyme alteration: a guide for clinicians. CMAJ 2005; 172: 367-379.
21. Shamban L, Patel B, Williams M. Significantly elevated liver alkaline phosphatase in congestive heart failure. Gastroenterol Res 2014; 7: 64-68.

22. Siddique A, Kowdley KV. Approach to a patient with elevated serum alkaline phosphatase. Clin Liver Dis 2012; 16: 199-229.

23. Bastis-Maounis B, Matsaniotis N, Maounis F. Serum alkaline phosphatase in infants with obstructive jaundice: relation to vitamin D supplementation. J Pediatr 1973; 82: 68-72.

24. Udomsinprasert W, Honsawek S, Anomasiri W, et al. Elevated adiponectin is associated with poor outcome in children with biliary atresia. Asian Biomed 2012; 6: 369-376.

25. Kasztelan-Szczerbinska B, Slomka M, Celinski K, et al. Alkaline phosphatase: the next independent predictor of the poor 90-day outcome in alcoholic hepatitis. Biomed Res Int 2013; 2013: 8.

26. Mayo MJ. Predicting outcomes of primary biliary cholangitis using the globe score. Gastroenterol Hepatol 2015; 11: 842-844.

27. Shteyer E, Ramm GA, Xu C, et al. Outcome after portoenterostomy in biliary atresia: pivotal role of degree of liver fibrosis and intensity of stellate cell activation. J Pediatr Gastroenterol Nutr 2006; 42: 93-99.

28. Ishak K, Baptista A, Bianchi L, et al. Histological grading and staging of chronic hepatitis. J Hepatol 1995; 22: 696-699.

29. Pape L, Olsson K, Petersen C, et al. Prognostic value of computerized quantification of liver fibrosis in children with biliary atresia. Liver Transplantation 2009; 15: 876-882.

30. Moyer K, Kaimal V, Pacheco C, et al. Staging of biliary atresia at diagnosis by molecular profiling of the liver. Genome Med 2010; $2: 33$. 